\title{
Nodular vasculitis or erythema induratum without cutaneous tuberculosis: An unusual presentation in an uncommon site
}

\author{
Ramin Mofarrah', Nafiseh Jallab², Ramina Mofarrah³, Kousar Jahani Amiri,, \\ Naghmeh Jallab ${ }^{4}$
}

\begin{abstract}
${ }^{1}$ Austria Board Certified Dermatologist and Venereologist, Assistant Professor, Department of Dermatology, Faculty of Medicine, Islamic Azad University of Medical Sciences, Sari Branch, Sari, Iran, ${ }^{2}$ General Physician, Islamic Azad University of Medical Sciences, Sari Branch, Sari, Iran, ${ }^{3}$ Medical Student, Student Research Committee, Shiraz University of Medical Sciences, Shiraz, Iran, ${ }^{4}$ Medical Student, Student Research Committee, Islamic Azad University of Medical Sciences, Sari Branch, Sari, Iran
\end{abstract}

Corresponding author: Dr. Ramin Mofarrah, E-mail: ramin.mofarrah@gmail.com

\begin{abstract}
Erythema induratum (EI) is a rare chronic disease, which occurs with cutaneous tuberculosis (TB). Nodular vasculitis, much rarer than erythema induratum, describes the same condition but without cutaneous TB, with lesions usually in the lower legs and rarely on the breasts. We report the case of a 46-year-old female with a history of crusted skin and necrotic lesions two years before, which, once self-limited, multiplied one month before and transferred to uncommon sites of the body, such as the breast. There was no evidence of other clinical presentations, and a chest X-ray gave no pathological findings. A biopsy was taken from the lesions, and the patient was diagnosed with erythema induratum without cutaneous tuberculosis, that is, nodular vasculitis. After treatment with calcineurin-inhibiting tacrolimus ointment, topical corticosteroids, and immunosuppressive oral azathioprine, the lesions improved.
\end{abstract}

Key words: Erythema induratum; Nodular vasculitis; Tuberculosis

\section{INTRODUCTION}

Nodular vasculitis (NV) is an uncommon, mostly lobar form of panniculitis [1], manifesting itself as erythematous nodules or plaques located preferentially on the calves, which may ulcerate and drain. NV has been thought of as a delayed hypersensitivity response to an antigenic stimulus, originally associated with tuberculosis. However, some cases have been associated with both nontuberculous infections, such as hepatitis B virus, hepatitis $\mathrm{C}$ virus, HIV, and streptococci, and noninfectious conditions [2], such as autoimmune diseases [3] and inflammatory bowel disease [1]. Chronic exposure to cold and drugs [4] have been reported. The term nodular vasculitis was coined by Montgomery to refer to cases of lesions similar to erythema induratum unassociated with tuberculosis [4]. These lesions are located on the anterior and/or posterior legs, including the calves, thighs, upper limbs, and trunk [5]. Erythema induratum (EI) has a chronic recurrent course and a female predominance, manifesting itself as persistent nodules or plaques usually on the lower third of the calf. Lesions may ulcerate and heal with a depressed scar. Histologically, a vasculitis affecting small and mediumsized vessels is seen producing a dermal granuloma with epithelioid and giant cells and fat atrophy [6].

\section{CASE REPORT}

We report the case of a 46-year-old female with a history of skin lesions two years before, which,

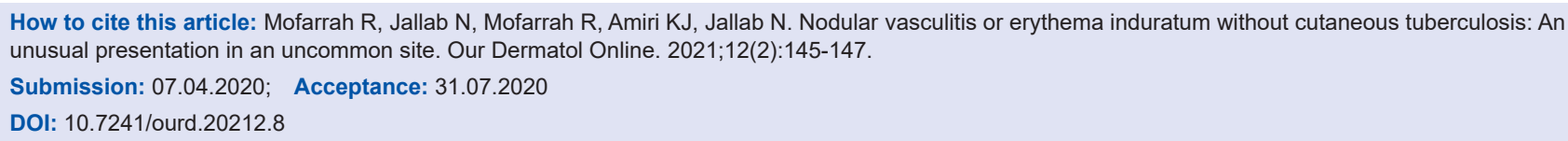


once self-limited, multiplied one month before and transferred with uncertain etiology. These firm, brownish-red to red lesions were located on the right breast and the posterior side of the left calf (Figs. la and $\mathrm{lb}$ ). The patient reported pain and pruritus in the lesions but no constitutional symptoms. She had a history of diabetes mellitus for two years. The physical examination and laboratory results were unremarkable. A tuberculin test was negative and a chest X-ray showed no pathological findings. A biopsy was taken from the breast and leg to show small fragments of skin with epidermal acanthosis and mild hyperkeratosis. Perivascular infiltration of inflammatory cells, mainly lymphocytes, and extravasation of red blood cells were present in the superficial and deep dermis. Vesicular lesions and dense infiltration of inflammatory cells were absent.

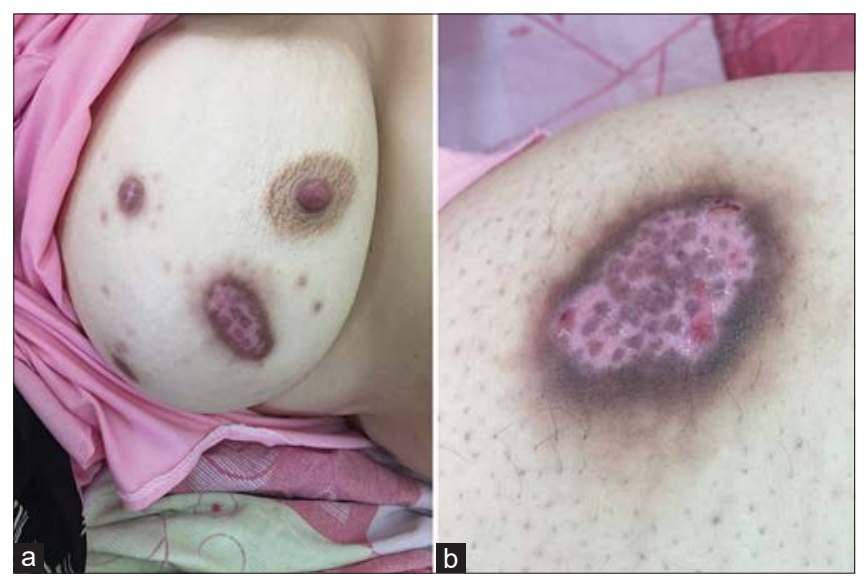

Figure 1: Hyperpigmented and erythematous ulcerated lesions on the left calve and right breast.
A granulomatous lesion with several multinucleated giant cells was also present in the deep dermis (Fig. 2). Based on these findings, a diagnosis of nodular vasculitis was reached. The patient was started on topical treatment with tacrolimus and clobetasol. After around two weeks, oral immunosuppression with azathioprine was started due to the lack of effectiveness of the previous treatment. This resulted in improvement of the lesions with no recurrence.

\section{DISCUSSION}

We report this case both because erythema induratum without $\mathrm{TB}$ is rare and because the breast is an uncommon site for this disease. Nodular vasculitis is a chronic relapsing lobular panniculitis with septal vasculitis. Its cause may be due to a hypersensitivity reaction to an antigenic stimulus [2]. Erythema induratum must be distinguished from erythema nodosum, nodular vasculitis, polyarteritis nodosa, tertiary syphilis, and other infectious and inflammatory panniculitides.

Erythema induratum was first identified in 1861 by Bazin as a nodular eruption on the posterior side of the lower legs in young women with tuberculosis [7]. In cases of erythema induratum with a negative tuberculin test, the cause is often unknown, but there have been case reports of an association of erythema induratum with infectious disorders caused by Nocardia, Pseudomonas spp., Fusarium spp., and the hepatitis $\mathrm{C}$ and $\mathrm{B}$ virus [3]. Erythema induratum,

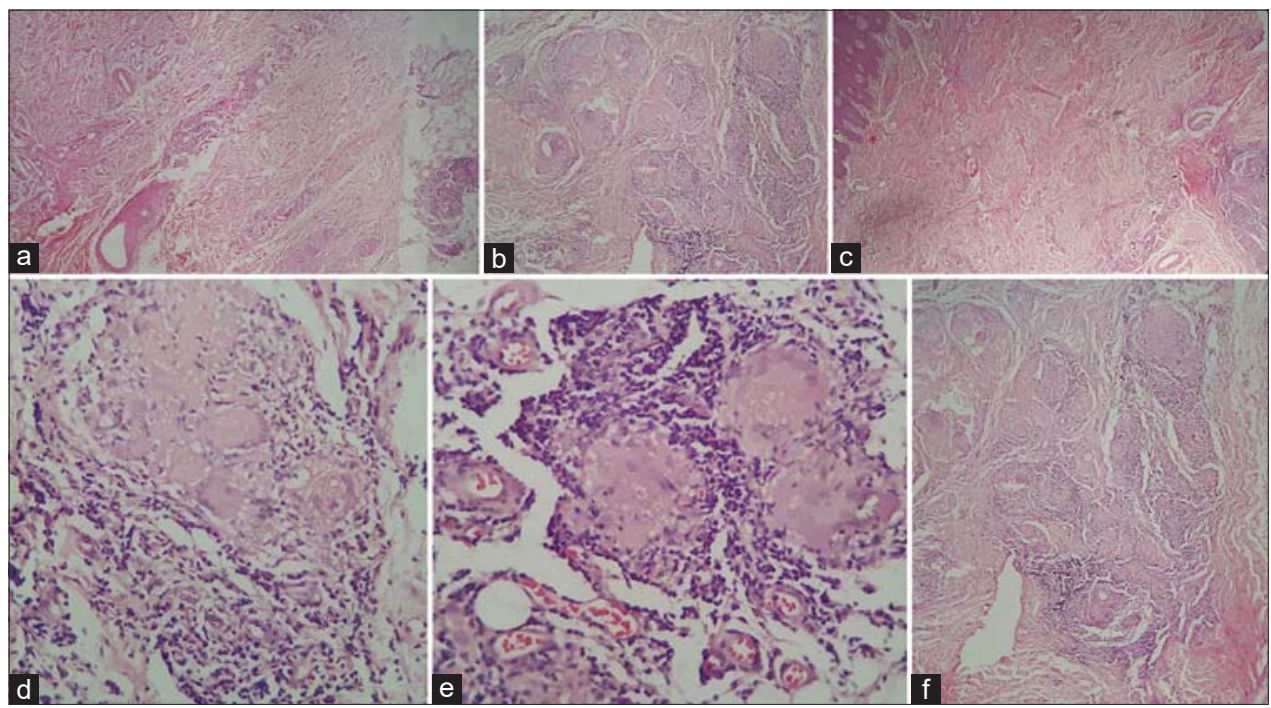

Figure 2: $(a-f)$ Histological studies of biopsy specimens showing epidermal acanthosis and mild hyperkeratosis; perivascular infiltration of inflammatory cells, mainly lymphocytes, and extravasation of red blood cells seen in the superficial and deep dermis; vesicular lesions and dense infiltration of inflammatory cells absent; a granulomatous lesion with several multinucleated giant cells in the deep dermis. 
one of the tuberculitides, affects males and females equally, although with a slight predominance among females in the age group of 20 to 40 years [8]. It manifests itself as tender, violaceous erythematous plaques or nodules characteristically on the bilateral pretibial areas. Lesions may also occur in other sites, such as the posterior and anterolateral pretibial sides of the lower legs as well as the trunk, buttocks, thighs, and arms $[8,9]$. Patients with erythema induratum have histories of tuberculosis and generally give significant results on the tuberculin test. Skin biopsies often show features of panniculitis with noncaseating granulomas. Tubercle bacilli are absent, although mycobacterial DNA is seen in up to $88 \%$ of erythema induratum cases. Although this condition is uncommon in developed countries, its incidence is still found to be high in countries such as India, Hong Kong, and South Africa [9]. It can occur in both sexes and at any age, but there is a female predominance usually seen through the second to fourth decade of life [8]. An examination of a skin biopsy from the lesional area revealed no tubercle bacilli, although mycobacterial DNA can be detected through the PCR in 56-88\% of patients with cutaneous tuberculosis. Despite that, a rare presence of atypical mycobacteria in the lesions of erythema induratum has been reported [10].

\section{CONCLUSION}

In conclusion, erythema induratum without tuberculosis (TB), known as nodular vasculitis, is a rare disease found in middle-aged women, which usually manifests itself as subcutaneous erythematous nodules and plaques appearing on the posterior side of the lower extremities. Our case was especially rare and unique, being unassociated with tuberculosis and displaying lesions in unusual sites, such as the breast.

\section{Consent}

The examination of the patient was conducted according to the principles of the Declaration of Helsinki.

The authors certify that they have obtained all appropriate patient consent forms, in which the patients gave their consent for images and other clinical information to be included in the journal. The patients understand that their names and initials will not be published and due effort will be made to conceal their identity, but that anonymity cannot be guaranteed.

\section{REFERENCES}

1. Misago N, Narisawa, Y. Erythema induratum (nodular vasculitis) associated Crohn's disease: a rare type of Crohn's disease. Am J Dermatopathol. 2012;34:325-9.

2. Borges AS, Brasileiro A, Apetato M. Nodular vasculitis associated with lung adenocarcinoma. An Bras Dermatol. 2018;93:887-9.

3. Gilchrist H, Patterson JW. Erythema nodosum and erythema induratum (nodular vasculitis): diagnosis and management. Dermatol Ther. 2010;23:320-7.

4. Park SB, Chang IK, Im M, Lee Y, Kim CD, Seo YJ, et al. Nodular vasculitis that developed during Etanercept (Enbrel) treatment in a patient with psoriasis. Ann Dermatol. 2015;27:605-7.

5. Chen Q, Chen WC, Hao F. Cutaneous tuberculosis: a great imitator. Clin in Dermatol. 2019;37:192-9.

6. Priya B, Smita J, Chandra AR. Concomitant erythema induratum of Bazin and papulonecrotic tuberculid: a rare manifestation. Our Dermatol Online. 2020;11:65-7.

7. Montgomery H, O'Leary PA, Barker NW. Nodular vascular disease of the legs. JAMA. 1945;128:335-41.

8. Gilchrist H, Patterson JW. Erythema nodosum and erythema induratum (nodular vasculitis): diagnosis and management. Dermatol Ther. 2010;23:320-7.

9. Nirmala C, Nagarajappa AH. Erythema induratum - a type of cutaneous tuberculosis. Indian J Tuberc. 2010;57:160-4.

10. Campbell SM, Winkelmann RR, Sammons DL. Erythema induratum caused by mycobacterium chelonei in an immunocompetent patient. J Clin Aesthet Dermatol. 2013;6:38-40.

Copyright by Ramin Mofarrah, et al. This is an open-access article distributed under the terms of the Creative Commons Attribution License, which permits unrestricted use, distribution, and reproduction in any medium, provided the original author and source are credited.

Source of Support: Nil, Conflict of Interest: None declared. 